\title{
n-3 Polyunsaturated fatty acids and immune function
}

\author{
Dayong Wu and Simin Nikbin Meydani* \\ Nutritional Immunology Laboratory, Jean Mayer USDA Human Nutrition Research Center on Aging at Tufts University, \\ Boston, MA 02111, USA
}

Considerable interest in fish oil was initially generated by epidemiological studies in Eskimos showing the beneficial effect of consuming fish and fish oil in preventing IHD (Dyerberg et al. 1978; Dyerberg \& Bang, 1979). In the following two decades, investigations of fish oil have been motivated by, and extended to, many different aspects of health and disease. A wide spectrum of studies has revealed the ability of fish oil to affect the course of cardiovascular disease, autoimmune and inflammatory diseases, immune function, infection, allograft rejection, and certain cancers (Fernandes \& Venkatraman, 1993; Calder, 1996).

The biological effects of fish oil are attributed to their $n-3$ polyunsaturated fatty acids (PUFA), mainly eicosapentaenoic acid (EPA) and docosahexaenoic acid (DHA). EPA and DHA can be incorporated into cell membranes where they influence membrane fluidity, receptor function, enzyme activity and production of lipid mediators.

\section{Polyunsaturated fatty acids and eicosanoid metabolism}

Changes in the fatty acid composition of the diet are known to modulate membrane phospholipid fatty acid composition (Huang et al. 1988; Clandinin et al. 1991), resulting in alteration in the oxygenated products of arachidonic acid (AA) (Davits \& Nugteren, 1988; Huang et al. 1988; Meydani et al. 1988; Knapp, 1990; Leaver et al. 1991). Some PUFA are the precursors of important lipid mediators; for example, dihomo- $\gamma$-linolenic acid, AA, and EPA respectively are immediate precursors for 1-, 2-, and 3-series prostaglandins (PG) in the cyclooxygenase (EC 1.14.99.1) pathway, and 3-, 4-, and 5-series leukotrienes (LT) in the lipoxygenase pathway. There is interaction among precursors, mainly through competitive incorporation into membrane lipids and competition for the common metabolizing enzymes. Eicosanoids originating from AA are the most abundant and also, in most cases, the most active mediators. $n-3$ PUFA can interfere with AA metabolism at the cyclooxygenase and lipoxygenase levels. Increased intake of $n$ - 3 PUFA decreases the generation of eicosanoids from AA (Huang et al. 1988; Meydani et al. 1988) and promotes the generation of the 3-series PG (Davits \& Nugteren, 1988; Knapp, 1990; Leaver et al. 1991) and 5-series LT (Lee et al. 1985; Leitch et al. 1985; Whelan et al. 1991).

\section{Polyunsaturated fatty acids, eicosanoids and immune function}

Immune cells have a high PUFA content in their membrane phospholipids. Immune cell functions are highly dependent on membrane-associated events. Interactions and cooperation of different cells of the immune system are regulated by the membrane-associated events through different protein and lipid mediators, which are essential in mounting a successful immune response. It is conceivable, therefore, that changes in membrane PUFA content will affect substrate availability in the formation of cyclooxygenase and lipoxygenase products which, in turn, act as lipid mediators that control the immune system (Goodwin \& Webb, 1980; RolaPlezczunski, 1985). Furthermore, the cells of the immune system perform their functions, in large part, through membrane-associated activities such as the secretion of cytokines and antibodies, antigen reception, lymphocyte transformation, and contact lysis, all of which can be affected by changes in membrane structure.

Immunity can be classified into two types, based on the components of the immune system that mediate the response, i.e. humoral immunity and cell-mediated immunity. Humoral immunity is mediated by molecules called antibodies that are produced by B lymphocytes and are responsible for specific recognition and elimination of extracellular antigens. Cell-mediated immunity is mediated by cells of the immune system, particularly T lymphocytes. Cell-mediated immunity is responsible for delayed-type hypersensitivity reactions, foreign graft rejection, resistance to many pathogenic micro-organisms, and tumour immunosurveillance. AA metabolites, including PG, LT, and hydroxyeicosatetraenoic acid, can be produced by immune cells in response to different stimuli. In general, cellular and humoral immune responses are negatively regulated by cyclooxygenase products. $\mathrm{PGE}_{2}$ has been shown to inhibit lymphocyte proliferation (Goodwin et al. 1974; Webb et al. 1980), cytokine production (Gordon et al. 1976), the generation of cytotoxic cells (Plaunt, 1979), and natural killer cell activity (Roder \& Klein, 1979). Lipoxygenase products of AA, i.e. LT and hydroxyeicosatetraenoic acid, also affect these immune functions (Goodman \& Weigle, 1980; RolaPlezczunski, 1985), although these controversial results have been presented in different studies.

\footnotetext{
Abbreviations: AA, arachidonic acid; ALA, $\alpha$-linolenic acid; Con A, concanavalin A; DHA, docosahexaenoic acid; EPA, eicosapentaenoic acid; IL, interleukin; LT, leukotriene; PBMC, peripheral-blood mononuclear cells; PG, prostaglandin; PHA, phytohaemagglutinin; PUFA, polyunsaturated fatty acids; TNF, tumour necrosis factor.
}

*Corresponding author: Dr Simin Nikbin Meydani, fax +1617556 3224, email S_Meydani_im@hnrc.tufts.edu 


\section{n-3 Polyunsaturated fatty acids and immune response}

$n-3$ PUFA, in addition to affecting general properties of cells as membrane components, play a role in modulating the production of both lipid (eicosanoids) and protein (cytokines) mediators. Administration of fish oil has been shown to suppress the production of pro-inflammatory eicosanoids ( $\mathrm{PGE}_{2}$ and $\mathrm{LTB}_{4}$ ) (Christophe \& Pascaud, 1990; Fritsche et al. 1992; Engstrom et al. 1996) and pro-inflammatory cytokines (interleukin (IL)-1, IL-6, tumour necrosis factor (TNF); Endres et al. 1989; Meydani et al. 1991a; Caughey et al. 1996), which may explain their beneficial effect on cardiovascular, autoimmune and inflammatory diseases. All these diseases are associated with a dysregulated immune response. Excessive levels of these mediators, which have often been demonstrated in such situations, can be suppressed by $n-3$ PUFA administration, resulting in attenuation of the disease process. However, such therapy often presents undesirable outcomes in healthy subjects or those with compromised immune status, since excessive reduction in cytokine or eicosanoid production could impair their normal host defence or homeostasis. Although some studies have demonstrated no significant effect (Berger et al. 1993), or even positive effect (Payan et al. 1986; Kelley et al. 1988; Fowler et al. 1993), on immune response by $n-3$ PUFA supplementation, a predominant number of studies have shown diminished cell-mediated immune function, such as lymphocyte proliferation, IL-2 production and delayed-type hypersensitivity, as a result of $n-3$ PUFA supplementation (Meydani et al. 1991a; Aki et al. 1992; Calder \& Newsholme, 1992; Endres et al. 1993; Jolly et al. 1997; Wander et al. 1997). In vitro supplementation with $n-3$ PUFA has also been observed to inhibit lymphocyte function in many studies in which lymphocytes from different sources were used and stimulated by a variety of agents (Calder, 1996). In addition, dietary fish oil has been shown to impair the resistance of mice to infection with Salmonella typhimurium (Chang et al. 1992) and Listeria monocytogenes (Fritsche et al. 1997). These undesirable effects compromise the beneficial potentials of $n-3$ PUFA as antiatherosclerotic and anti- inflammatory compounds. However, studies indicate that it might be possible to establish fish oil supplementation protocols that provide maximal beneficial effects and minimal adverse effects. The present article will review a series of studies conducted by our laboratory, examining the immunological effect of $n-3$ PUFA in both human subjects and animals.

\section{The effect of oral $n-3$ polyunsaturated fatty acid supplementation on cytokine production and lymphocyte proliferation in young and older women}

Many studies have indicated a beneficial effect of $n$-3 PUFA supplementation on atherosclerosis and atherothrombotic disorders, and inflammatory diseases. The prevalence of all these disorders increases with age. Ageing is associated with an altered regulation of the immune system (Siskind, 1980). Age-associated functional changes have been well characterized for both humoral and cell-mediated immune responses. However, the age-associated changes are mostly observed in T-cell-mediated functions (Miller, 1994). Thus, we determined the effect of $n-3$ PUFA supplementation on the immune response of young and older subjects (Meydani et al. 1991a).

Six healthy young (23-33 years) and six healthy older (51-68 years) women were recruited after they were screened for their disease history, health status and drug use. Each subject's usual diet was supplemented for 3 months with n-3 PUFA contained in Pro-Mega capsules (Parke Davis, Warner Lambert Co., Morris Plains, NJ, USA), providing $1.68 \mathrm{~g}$ EPA, $0.72 \mathrm{~g}$ DHA, $0.6 \mathrm{~g}$ other fatty acids and $6 \mathrm{mg}$ vitamin E/d. Blood was collected at baseline and at the end of 1,2 and 3 months of supplementation with fish oil. Peripheral-blood mononuclear cells (PBMC) were separated from blood and cultured in the presence or absence of mitogens for measurement of lymphocyte proliferation, cytokine and $\mathrm{PGE}_{2}$ production.

The results showed that $n$-3 PUFA supplementation for 3 months significantly increased plasma levels of EPA and DHA in both young and older subjects; however, the increase in EPA and DHA was greater in older subjects than in young subjects (10-fold increase for EPA and 2.5-fold increase for DHA in older subjects $v$. 5-fold increase for EPA and 1.6-fold increase for DHA in young subjects). AA was significantly decreased in older subjects, whereas it did not change in young subjects, resulting in a lower value for AA : EPA in older subjects than in young subjects. There was no significant age-associated difference in the production of inflammatory cytokines. Supplementation with $n-3$ PUFA decreased the production of all the inflammatory cytokines tested in both young and older subjects, with a greater decrease being observed in older subjects: IL-1 $\beta$ synthesis was reduced by $48 \%$ in young subjects and $90 \%$ in older subjects; TNF was reduced by $58 \%$ in young and $70 \%$ in older subjects; IL-6 was reduced by $30 \%$ in young and 60 $\%$ in older subjects. IL-2, a T-cell growth factor necessary for lymphocyte activation, was produced in a significantly lower amount in older subjects than in young subjects at baseline and after 1 month of $n-3$ PUFA supplementation. Consumption of $n-3$ PUFA tended to reduce IL-2 production, but only the reduction in older subjects reached significance. Proliferative response of PBMC to T-cell mitogens was significantly lower in older subjects at all time points compared with that in young subjects. Supplementation with $n-3$ PUFA inhibited lymphocyte proliferation significantly in older subjects (36\% reduction after 3 months) but not in young subjects. $\mathrm{PGE}_{2}$, a suppressive factor of T-cell function, was found to be decreased by $57 \%$ in older subjects and $40 \%$ in young subjects after 3 months of $n$-3 PUFA supplementation. However, the decrease seen in young subjects did not reach statistical significance. Similar results were observed by Endres et al. $(1989,1993)$, who demonstrated that $n$-3 PUFA supplementation $(4.69 \mathrm{~g} / \mathrm{d})$ in healthy men for 6 weeks reduced IL-1, TNF and IL-2 production, and lymphocyte proliferation.

It is interesting to note the age-associated difference in the suppressive effect of $n-3$ PUFA on these variables. While the mechanism of this age difference was not determined, the more dramatic changes in older subjects were associated with a larger increase in plasma EPA and DHA and a greater decrease in AA in older subjects compared with young subjects. This difference in the magnitude of $n$ - 3 PUFA-induced 
changes in plasma fatty acid might be a result of ageassociated differences in fatty acid absorption (Hollander $e t$ al. 1984).

$\mathrm{PGE}_{2}$ has been shown to suppress IL-1 and IL-2 production, and lymphocyte proliferation (Goodwin \& Webb, 1980; Knusden et al. 1986). We showed that a decrease in $\mathrm{PGE}_{2}$ production by $\alpha$-tocopherol supplementation enhances IL-2 production and lymphocyte proliferation in old mice (Meydani et al. 1986) and elderly human subjects (Meydani et al. 1990). Contrary to the presumption that reduced production of $\mathrm{PGE}_{2}$ should lead to enhanced IL-2 production and lymphocyte proliferation, $n$-3 PUFA supplementation resulted in suppressed T-cell-mediated function. Similarly, Meydani et al. (1988) showed a decrease in both $\mathrm{PGE}_{2}$ production and natural killer cell activity in mice fed on a fish oil-supplemented diet, although $\mathrm{PGE}_{2}$ has been reported to inhibit natural killer cell activity (Roder \& Klein, 1979; Brunda et al. 1980). These results indicate that mechanisms other than those mediated through $\mathrm{PGE}_{2}$ may exist and play a more dominant role in the $n-3$ PUFA-induced effect on immune function. Excessive production of lipid peroxides such as $\mathrm{H}_{2} \mathrm{O}_{2}$ has been shown to suppress lymphocyte proliferation (Zoschke \& Messner, 1984). Meydani et al. (1991b) showed that $n$ - 3 PUFA supplementation increased plasma malonaldehyde levels, and this increase was greater in older women than in young women. IL-2 is a key cytokine in cell-mediated immune response, and its production may be affected directly by PUFA, independent of the changes in eicosanoid production, as indicated by Santoli \& Zurier (1989). Furthermore, Hughes and colleagues showed, in both in vivo (Hughes et al. 1996a) and in vitro (Hughes \& Pinder, 1996; Hughes et al. 1996b) studies, that $n-3$ PUFA supplementation can inhibit the antigen-presenting function of human blood monocytes by reducing the expression of related cell surface molecules such as major histocompatibility complex class II, intercellular adhesion molecule-1, leukocyte-function-associated antigens-1 and -3. A recent study (Jolly et al. 1997) showed that feeding mice on a low-fat diet enriched in $n$ - 3 PUFA (EPA and DHA) for a short period $(10 \mathrm{~d})$ suppressed concanavalin A (Con A)-induced lymphocyte proliferation and IL-2 production, which was accompanied by a reduction in the production of lipid second messengers, diacylglycerol and ceramide. Diacylglycerol and ceramide play an important role in murine T-cell proliferation (Jolly et al. 1996). In vitro supplementation with EPA and DHA was also shown to inhibit protein kinase activity in mouse splenocytes (VanMeter et al. 1994) and in rat peritoneal macrophages (Tappia et al. 1995). These results indicate that the $n-3$ PUFA-induced effects on T-cells might be mediated through a change in signal transduction. Further discussion of this mechanism is provided later (see pp. 505-506).

\section{Immunological effect of long-term feeding of low-fat, low-cholesterol diets with or without fish-derived $n-3$ polyunsaturated fatty acid enrichment}

In order to determine if the suppressive effects observed were specific to $n-3$ PUFA or not, we investigated the effect of long-term feeding of the National Cholesterol Education Panel Step-2 diet high in fish-derived $n-3$ PUFA or in plant- derived $n-6$ and $n-3$ PUFA on the immune response of healthy adults (Meydani et al. 1993). Twenty-two healthy volunteers over the age of 40 years were divided into two groups and fed on National Cholesterol Education Panel Step-2 diets enriched with either $1.23 \mathrm{~g} \mathrm{EPA}$ and DHA/d (high-fish) or $0.27 \mathrm{~g} \mathrm{EPA}$ and DHA/d (low-fish); the remaining $n-3$ PUFA in the two diets was provided as plant-derived linolenic acid, $18: 3 n-3$. After 6 months of supplementation, the high-fish group had a small but significant decrease in the percentage of helper T-cells, whereas the percentage of suppressor $\mathrm{T}$-cells was increased. This change was accompanied by a significant reduction in delayed-type hypersensitivity skin response and proliferative response of PBMC to T-cell mitogen Con A. A significant correlation between changes in delayed-type hypersensitivity and plasma EPA levels was observed. As reported in previous studies, IL-1 $\beta$, TNF- $\alpha$ and IL- 6 produced by PBMC from subjects fed on the high-fish diet were significantly reduced. In addition, a non-significant decrease in IL-2 and granulocyte macrophage growth factor was observed in this group. In contrast, the low-fish group showed increased PBMC proliferative response to Con $A$ as well as production of IL- $1 \beta$ and TNF- $\alpha$, but no significant effect on delayedtype hypersensitivity, IL-6, granulocyte macrophage growth factor or $\mathrm{PGE}_{2}$ production. These results indicated that the suppressive effect of $n-3$ PUFA is not observed with plantderived $n-6$ and $n-3$ PUFA. When expressed relative to PUFA (per double bonds), plasma $\alpha$-tocopherol was significantly decreased in the high-fish group but not in the low-fish group. Since a decrease in $\alpha$-tocopherol level has been shown to suppress T-cell-mediated function, these results indicate that $n-3$ PUFA may in part exert their effect indirectly through a reduction in $\alpha$-tocopherol level. Reduction in tocopherol status has also been observed following fish oil consumption in animals (Meydani et al. 1987; Fritsche et al. 1992; Farwer, 1994; Wander et al. 1997).

\section{Studies on the mechanism of immuno-suppressive effect of $\boldsymbol{n - 3}$ polyunsaturated fatty acid supplementation in mice}

As discussed previously, decreased $\mathrm{PGE}_{2}$ production after $n-3$ PUFA consumption does not result in an enhanced immune response as expected; rather, $n-3$ PUFA in most studies were shown to suppress immune response. Increased lipid peroxidation (Piche et al. 1988; L'Abbe et al. 1991; Meydani et al. 1991b; Wander et al. 1996, 1997) and compromised vitamin E status (Meydani et al. 1987; Fritsche et al. 1992; Farwer, 1994; Wander et al. 1997) have been implicated as contributing factors to the immunological effects of marine-derived $n-3$ PUFA. To strengthen the evidence for this hypothesis, and to further explore the role of eicosanoids, we conducted the following studies (Shapiro et al. 1993, 1994).

Specific pathogen-free C57BL/6NIA mice ( 2 months old) were randomly assigned to two groups (twenty-four per group) and fed on semi-purified diets containing either high levels of $n-3$ PUFA ( $88 \mathrm{~g}$ fish oil $+12 \mathrm{~g}$ maize oil $/ \mathrm{kg}$ ) or $n-6$ PUFA ( $70 \mathrm{~g}$ maize oil $+30 \mathrm{~g}$ lard $/ \mathrm{kg}$ ) for 8 weeks. The mice were killed at the end of the supplementation period. Tissue fatty acid and vitamin $\mathrm{E}$ levels were measured, and 
splenocytes were isolated to be used for in vitro immune response analyses. $n$-3 PUFA-fed mice showed a significant increase in EPA and DHA, and a decrease in AA of total liver fatty acids. Liver vitamin $\mathrm{E}$ levels were lower in the $n-3$ PUFA group compared with the $n-6$ PUFA group. A significant decrease in the percentage of total T-cells, $\mathrm{T}$ helper and $\mathrm{T}$ suppressor cells, and macrophages was observed in the spleens from $n-3$ PUFA-fed mice. Associated with these changes, a suppressed mitogenic response of splenocytes to phytohaemagglutinin (PHA) was also observed in $n-3$ PUFA-fed mice. Next, splenocytes were cultured in the presence of different levels of $\mathrm{AA}\left(\mathrm{PGE}_{2}\right.$ and $\left.\mathrm{LTB}_{4}\right)$ - and EPA $\left(\mathrm{PGE}_{3}\right.$ and $\left.\mathrm{LTB}_{5}\right)$-derived eicosanoids or vitamin $\mathrm{E}$. While both $\mathrm{PGE}_{2}$ and $\mathrm{PGE}_{3}$ were found to inhibit PHAinduced splenocyte proliferation, relatively higher concentrations of $\mathrm{PGE}_{2}$ were needed to obtain the same percentage inhibition of proliferation than were needed for $\mathrm{PGE}_{3}$. This finding indicated that $\mathrm{PGE}_{3}$ has the same or a greater suppressive effect on lymphocyte proliferation than $\mathrm{PGE}_{2}$. In vitro addition of $\mathrm{LTB}_{4}$ did not significantly affect splenocyte proliferation induced by Con $\mathrm{A}$ or PHA, whereas addition of $\mathrm{LTB}_{5}$ tended to inhibit Con A-induced proliferation of splenocytes from $n-3$ PUFA-fed mice. Similar results were observed in another study in which in vitro addition of $\mathrm{PGE}_{3}$ and $\mathrm{LTB}_{5}$ diminished Con $\mathrm{A}$-induced mitogenic response in human PBMC to a larger extent than the same concentrations of $\mathrm{PGE}_{2}$ and $\mathrm{LTB}_{4}$ (Shapiro et al. 1993). Thus, while $n-3$ PUFA decrease formation of $\mathrm{PGE}_{2}$, the production of $\mathrm{PGE}_{3}$ and $\mathrm{LTB}_{5}$ following the consumption of $n-3$ PUFA could contribute to the suppression of lymphocyte proliferation. Vitamin $\mathrm{E}$ added in vitro significantly enhanced lymphocyte proliferation in both control and fish oil-fed mice; however, vitamin $E$ induced a larger increase in mice fed on the $n-3$ PUFA diet (281\%) than in those fed on the $n$ - 6 PUFA diet $(109 \%)$. This is consistent with the studies reported by Kramer et al. (1991) in which supplementation of human subjects with $15 \mathrm{~g}$ fish oil/d for 10 weeks suppressed the mitogenic responsiveness of PBMC to Con A, and supplementation with $200 \mathrm{mg}$ tocopherol for 8 weeks reversed the depressed mitogenic response induced by feeding fish oil. They further showed a positive correlation between plasma $\alpha$-tocopherol concentrations and responsiveness of PBMC to Con A.

Taken together, these results indicate that increased production of EPA-derived eicosanoids and decreased levels of vitamin $E$ may contribute to the immuno-suppression observed in $n-3$ PUFA-supplemented human subjects and animals.

\section{Comparison of immunological effects of marine- and plant-derived $n-3$ polyunsaturated fatty acids in non-human primates}

To further determine the role of vitamin $\mathrm{E}$ and increased lipid peroxidation in $n-3$ PUFA-induced alteration of immune response, the following experiment was conducted in non-human primates (Wu et al. 1996). In this study, the immunological effects of feeding two sources of $n-3$ PUFA, i.e. marine- and plant-derived, in the presence of adequate levels of tocopherol was determined. The plant-derived $n-3$ PUFA were included because they, particularly $\alpha$-linolenic acid (ALA, 18:3n-3), have been indicated to provide the same benefit as supplementation with fish oil, but with fewer adverse effects (Simopoulos et al. 1986; Hunter, 1987); their effect on immune response was not well documented.

Twenty male cynomolgus monkeys (Macaca fascicularis) were fed on a baseline diet containing $30 \%$ of energy as fat for 14 weeks. They were then randomly divided into two groups (ten per group). One group was fed on a diet containing $3.5 \%$ energy as ALA for 14 weeks, followed by a diet containing $5.3 \%$ energy as ALA for another 14 weeks. The other group was fed on diets containing EPA and DHA (combined) at $1.3 \%$ energy and at $3.3 \%$ energy, each for 14 weeks. The other fatty acids were similar in all diets, and the amount of tocopherol added to the diets was calculated according to the mathematical formula described by Muggli (1989). Previous studies were confounded by the fact that either adequate tocopherol was added to the $n$ - 3 PUFA, or the same amount of tocopherol was added to the $n$-3 PUFA diet and the control diet, often a less-unsaturated diet. Blood samples were collected at the end of each dietary period for laboratory analysis.

The results showed that plasma fatty acid profiles generally reflected the fatty acid compositions of the respective dietary lipid sources. Mitogenic response of PBMC to Con A and PHA were significantly enhanced by 51 and $47 \%$ respectively after consumption of EPA and DHA at $3.3 \%$ energy, while no significant change was observed in the ALA group. Similarly, both Con A- and PHA-stimulated IL-2 production by PBMC was significantly increased after consumption of EPA and DHA but not ALA. Con Astimulated IL-2 production was promoted by 111 and $128 \%$ respectively after consumption of diets containing 1.3 and $3.3 \%$ energy as EPA and DHA, and also a $140 \%$ increase in PHA-stimulated IL-2 production was observed after consumption of the diet containing $3.3 \%$ energy as EPA and DHA. Consumption of both EPA and DHA and ALA significantly inhibited $\mathrm{PGE}_{2}$ production by $\mathrm{PBMC}$ on stimulation by Con $\mathrm{A}$ or PHA, with a higher degree of inhibition seen in the EPA and DHA group than in the ALA group. The lymphocyte subpopulations, as analysed by flow cytometry, showed a reduction in the percentage of T-cells, $T$ helper cells, and T suppressor cells after consumption of both EPA and DHA and ALA diets, which is consistent with our previous finding in mice (Shapiro et al. 1994). In this study, $\alpha$-tocopherol was added to the diets based on the existing formulas (Muggli, 1989). Accordingly, no significant change in $\alpha$-tocopherol level was observed following EPA and DHA consumption. Unexpectedly, however, plasma $\alpha$-tocopherol concentrations significantly decreased by $19 \%$ and $27 \%$ respectively after consumption of the diets containing 3.5 and $5.3 \%$ energy as ALA. This inadequate supply of vitamin $E$ was further reflected in the plasma phosphatidylcholine hydroperoxide concentration adjusted for the unsaturated index (per double bond). ALA-fed animals tended to have a higher plasma phosphatidylcholine hydroperoxide production than did those fed on EPA and DHA. Thus, it appears that the calculated $\alpha$-tocopherol requirement based on the amount of unsaturation of oils in the diets (Muggli, 1989) may not adequately reflect the actual levels needed in vivo when fatty acids have the potential to be further elongated and desaturated in the body to 
more unsaturated PUFA, as demonstrated by the existence of EPA and DHA in ALA-fed animals. The change in vitamin $\mathrm{E}$ status and hydroperoxide production may have offset the immune-enhancing effect that could otherwise have resulted from inhibited $\mathrm{PGE}_{2}$ production caused by ALA consumption.

The results of this study further support the importance of adequate vitamin E levels when the effect of $n$-3 PUFA on immune response is determined. In addition, this study demonstrated that the suppressive effect of $n$-3 PUFA administration on T-cell function can be prevented by vitamin E supplementation. Our current studies are designed to determine the levels of $\alpha$-tocopherol needed to maintain T-cell-mediated immune competence and the oxidantantioxidant balance in old subjects while preserving the anti-inflammatory effects of $n-3$ PUFA.

\section{Summary}

$n$-3 PUFA have been shown to reduce the risk of cardiovascular and inflammatory diseases. However, they have also been shown to suppress T-cell-mediated immune function, an undesirable effect, especially in immuno-suppressed individuals. Studies have thus far suggested that this immuno-suppression may be in part attributable to increased lipid peroxidation and decreased antioxidant (especially vitamin E) levels, which can be prevented by appropriate vitamin $\mathrm{E}$ supplementation. Further well-designed human studies are needed to determine the appropriate levels of $n-3$ PUFA and vitamin E supplementation to optimize the beneficial anti-inflammatory effect of $n-3$ PUFA and minimize their suppressive effect on T-cell function.

\section{References}

Aki H, Morinaga SI, Yamazaki K, Suzuki H \& Nakamura N (1992) Reduction of delayed-type hypersensitivity by the injection of $n-3$ polyunsaturated fatty acid in mice. Transplantation 54, 511-514.

Berger A, German JB, Chiang BL, Ansari AA, Keen CL, Fletcher MP \& Gershwin ME (1993) Influence of feeding unsaturated fats on growth and immune status of mice. Journal of Nutrition 123, 225-233.

Brunda MJ, Heberman RB \& Holden HT (1980) Inhibition of natural killer cell activity by prostaglandins. Journal of Immunology 124, 2682-2687.

Calder PC (1996) Immunomodulatory and anti-inflammatory effects of $n-3$ polyunsaturated fatty acids. Proceedings of the Nutrition Society 55, 737-774.

Calder PC \& Newsholme EA (1992) Polyunsaturated fatty acids suppress human peripheral blood lymphocyte proliferation and interleukin-2 production. Clinical Science 37, 695-700.

Caughey GE, Mantzioris E, Gibson RA, Cleland LG \& James MJ (1996) The effect on human tumor necrosis factor $\alpha$ and interleukin $1 \beta$ production of diets enriched in $n-3$ fatty acids from vegetable oil or fish oil. American Journal of Clinical Nutrition 63, 116-122.

Chang HR, Dulloo AG, Vladoianu IR, Piguet PF, Arsenijevic D, Girardier L \& Pechere JC (1992) Fish oil decreases natural resistance of mice infection with Salmonella typhimurium. Metabolism 41, 1-2.
Christophe B \& Pascaud M (1990) Effects of moderate dietary supplementations with $n-3$ fatty acids on macrophage eicosanoid synthesis in the rat. Biochimica et Biophysica Acta 1047, 19-28.

Clandinin MT, Cheema S, Field CJ, Garg ML, Venkatraman J \& Clandinin TR (1991) Dietary fat: exogenous determination of membrane structure and cell function. FASEB Journal 5 , 2761-2769.

Davits FA \& Nugteren A (1988) The urinary excretion of prostaglandin $\mathrm{E}$ and their corresponding tetranor metabolites by rats fed a diet rich in eicosapentaenoate. Biochimica et Biophysica Acta 958, 289-299.

Dyerberg J \& Bang HO (1979) Haemostatic function and platelet polyunsaturated fatty acids in Eskimos. Lancet ii, 433-435.

Dyerberg J, Bang HO, Stoffersen E, Moncada S \& Vane J (1978) Eicosapentaenoic acid and prevention of thrombosis and atherosclerosis. Lancet ii, 117-119.

Endres S, Ghorbani R, Kelley VE, Georgilis K, Lonneman G, Van der Meer WM, Cannon JG, Rogers TS, Klempner MS, Webber PC, Schaefer EJ, Wolf SM \& Dinarello DA (1989) The effect of dietary supplementation with $(n-3)$ polyunsaturated fatty acids on the synthesis of interleukin-1 and tumor necrosis factor by mononuclear cells. New England Journal of Medicine 320, 265-271.

Endres S, Meydani SN, Ghorbani R, Schindler R \& Dinarello CA (1993) Dietary $n$-3 fatty acids suppress interleukin-2 production and mononuclear cell proliferation. Journal of Leukocyte Biology 54, 599-603.

Engstrom K, Luostarinen R \& Saldeen T (1996) Whole blood production of thromboxane, prostacyclin and leukotriene $\mathrm{B}_{4}$ after dietary fish oil supplementation in man: effect of vitamin $E$. Prostaglandins, Leukotrienes and Essential Fatty Acids 54, $419-425$.

Farwer SR (1994) The vitamin E nutritional status of rats fed on diets high in fish oil, linseed oil, or sunflower seed oil. British Journal of Nutrition 72, 127-145.

Fernandes G \& Venkatraman JT (1993) Role of omega-3 fatty acids in health and disease. Nutrition Research 13, S19-S45.

Fowler KH, Chapkin RS \& McMurray DN (1993) Effects of purified dietary $n-3$ ethyl esters on murine T lymphocyte function. Journal of Immunology 151, 5186-5197.

Fritsche KL, Cassity NA \& Huang S-C (1992) Dietary (n-3) fatty acid and vitamin $E$ interactions in rats: effects on vitamin $E$ status, immune cell prostaglandin E production and primary antibody response. Joumal of Nutrition 122, 1009-1018.

Fritsche KL, Shahbazian LM, Feng C \& Berg JN (1997) Dietary fish oil reduces survival and impairs bacterial clearance in $\mathrm{C} 3 \mathrm{H} /$ Hen mice challenged with Listeria monocytogenes. Clinical Science 92, 95-101.

Goodman MG \& Weigle WO (1980) Modulation of lymphocyte activation I. Inhibition of an oxidation product of arachidonic acid. Journal of Immunology 125, 593-600.

Goodwin JS, Messner RP \& Peake GT (1974) Prostaglandin suppression of mitogen stimulated leukocytes in culture. Journal of Clinical Investigation 4, 368-378.

Goodwin JS \& Webb DR (1980) Regulation of the immune response by prostaglandins. Clinical Immunology and Immunopathology 15, 106-122.

Gordon D, Bray M \& Morley J (1976) Control of lymphokine secretion by prostaglandins. Nature 262, 401-402.

Hollander D, Dadufabza VD \& Sletten EG (1984) Does essential fatty acid absorption change with age? Journal of Lipid Research 25, 129-134.

Huang DH, Boudreau M \& Chanmugam P (1988) Dietary linolenic acid and longer-chain $n-3$ fatty acids: comparison of effects on arachidonic acid metabolism in rats. Journal of Nutrition 118, $427-437$. 
Hughes DA \& Pinder AC (1996) Influence of $n-3$ polyunsaturated fatty acids (PUFA) on the antigen-presenting function of human monocytes. Biochemical Society Transactions 24, 389S.

Hughes DA, Pinder AC, Piper Z, Johnson IT \& Lund EK (1996a) Fish oil supplementation inhibits the expression of major histocompatibility complex class II molecules and adhesion molecules on human monocytes. American Journal of Clinical Nutrition 63, 267-272.

Hughes DA, Southon S \& Pinder AC (1996b) (n-3) Polyunsaturated fatty acids modulate the expression of functionally associated molecules on human monocytes in vitro. Journal of Nutrition 126, 603-610.

Hunter JE (1987) Correspondence: More on fish oil (letter). New England Journal of Medicine 316, 626.

Jolly CA, Jiang Y-H, Chapkin RS \& McMurray DN (1997) Dietary $(n-3)$ polyunsaturated fatty acids suppress murine lymphoproliferation, interleukin-2 secretion, and the formation of diacylglycerol and ceramide. Journal of Nutrition 127, 37-43.

Jolly CA, Laurenz JC, McMurray DN \& Chapkin RS (1996) Diacylglycerol and ceramide kinetics in primary cultures of activated T-lymphocytes. Immunology Letters 49, 43-48.

Kelley DS, Nelson GJ, Serrato CM, Schmidt PC \& Branch LB (1988) Effects of type of dietary fat on indices of immune status of rabbits. Journal of Nutrition 118, 1376-1384.

Knapp HR (1990) Prostaglandins in human semen during fish oil ingestion: evidence for in vivo cyclooxygenase inhibition and appearance of novel trienoic compound. Prostaglandins 39 , 407-423.

Knusden PJ, Dinarello CA \& Strom TB (1986) Prostaglandins post-transcriptionally inhibit monocyte expression of interleukin 1 activity by increasing intracellular cyclic adenosine monophosphate. Journal of Immunology 137, 3189-3194.

Kramer TR, Schoene N, Dougless LW, Judd JT, Ballard-Barbash R, Taylor PR, Bhagavan N \& Nair PP (1991) Increased vitamin $E$ intake restores fish oil-induced suppressed blastogenesis of mitogenic-stimulated $\mathrm{T}$ lymphocytes. American Journal of Clinical Nutrition 54, 896-902.

L'Abbe MR, Trick KD \& Beare-Rogers J (1991) Dietary ( $n-3)$ fatty acids affect rat heart, liver and aorta protective enzyme activities and lipid peroxidation. Journal of Nutrition 121, 1331-1340.

Leaver HA, Howie A \& Wilson NH (1991) The biosynthesis of the 3 -series prostaglandins in rat uterus after alpha-linolenic acid feeding, mass spectroscopy of prostaglandins $E$ and $F$ produced by rat uteri in tissue culture. Prostaglandins, Leukotrienes and Essential Fatty Acids 42, 217-224.

Lee TH, Hoover R, Williams JD, Sperling J, Raralese J, Spur BW, Robinson DR, Corey EJ, Lewis RA \& Austen KF (1985) Effect of dietary enrichment with eicosapentaenoic and docosahexaenoic acids on in vitro neutrophil and monocyte leukotriene generation and neutrophil function. New England Journal of Medicine 312, 1217-1224.

Leitch AG, Lee TH, Ringel EW, Prickett JD, Robinson WR, Pyme SG, Corey EJ, Drazen JM, Austen KF \& Lewis RA (1985) Immunologically induced generation of tetraene and pentaene leukotrienes in the peritoneal cavities of menhaden-fed rats. Journal of Immunology 132, 2559-2565.

Meydani M, Natiello F, Goldin B, Free N, Woods M, Schaefer E, Blumberg JB \& Gorbach SL (1991b) Effect of long-term fish oil supplementation on vitamin $E$ status and lipid peroxidation in women. Journal of Nutrition 121, 484-491.

Meydani SN, Barklund MP, Liu S, Meydani M, Miller RA, Cannon JG, Morrow FD, Rocklin R \& Blumberg JB (1990) Vitamin E supplementation enhances cell-mediated immunity in healthy elderly subjects. American Journal of Clinical Nutrition 52, 557-563.
Meydani SN, Endres S, Woods MN, Goldin RD, Soo C, Morrill-Labrode A, Dinarello CA \& Gorbach SL (1991a) Oral $(n-3)$ fatty acid supplementation suppresses cytokine production and lymphocyte proliferation: Comparison between young and older women. Journal of Nutrition 121, 547-555.

Meydani SN, Lichtenstein AH, Cornwall S, Meydani M, Goldin BR, Rasmussen H, Dinarello CA \& Schaefer E (1993) Immunologic effects of National Cholesterol Education Panel (NCEP) Step-2 diets with and without fish-derived $n-3$ fatty acid enrichment. Journal of Clinical Investigation 92, 105-113.

Meydani SN, Meydani M, Verdon CP, Shapiro AC, Blumberg JB \& Hayes KC (1986) Vitamin E supplementation suppresses prostaglandin $\mathrm{E}_{2}$ synthesis and enhances the immune response of aged mice. Mechanisms of Ageing and Development 34, 191-201.

Meydani SN, Shapiro AC, Meydani M, Macauley JB \& Blumberg JB (1987) Effect of age and dietary fat (fish, corn and coconut oils) on tocopherol status of C57BL/6NIA mice. Lipids 22, 345-350.

Meydani SN, Yogeeswaran G, Liu S, Baskar S \& Meydani M (1988) Fish oil and tocopherol-induced changes in natural killer cell-mediated cytotoxicity and $\mathrm{PGE}_{2}$ synthesis in young and old mice. Journal of Nutrition 118, 1245-1252.

Miller RA (1994) Aging and immune function, cellular and biochemical analyses. Experimental Gerontology 29, 21-35.

Muggli R (editor) (1989) Dietary fish oils increase the requirement for vitamin $E$ in humans. St John's, Nfld., Canada: Biochemical Publishers \& Distributors.

Payan DG, Wong MYS \& Chernov-Rogan T (1986) Alterations in human leukocyte function induced by ingestion of eicosapentaenoic acid. Journal of Clinical Immunology 6, 402-410.

Piche LA, Draper HH \& Cole PD (1988) Malondialdehyde excretion by subjects consuming cod liver oil versus a concentrate of $n-3$ fatty acids. Lipids $23,370-371$.

Plaunt M (1979) The role of cyclic AMP in modulating cytotoxic T lymphocytes. Journal of Immunology 123, 692-701.

Roder JC \& Klein M (1979) Target-effector interaction in the natural killer cell system. Journal of Immunology 123, 2785-2790.

Rola-Plezczunski M (1985) Immunoregulation by leukotrienes and other lipoxygenase metabolites. Immunology Today 6, 302-307.

Santoli D \& Zurier RB (1989) Prostaglandin E precursor fatty acids inhibit human IL-2 production by a prostaglandin E-dependent mechanism. Journal of Immunology 143, 1303-1309.

Shapiro AC, Wu D, Hayek MG, Meydani M \& Meydani SN (1994) Role of eicosanoids and vitamin $\mathrm{E}$ in fish oil-induced changes of splenocyte proliferation to $\mathrm{T}$ cell mitogens in mice. Nutrition Research 14, 1339-1354.

Shapiro AC, Wu D \& Meydani SN (1993) Eicosanoids derived from arachidonic and eicosapentaenoic acids inhibit $\mathrm{T}$ cell proliferative response. Prostaglandins 45, 229-240.

Simopoulos AP \& Salem N (1986) Purslane: a terrestrial source of omega-3 fatty acids. New England Journal of Medicine 315, 833.

Siskind GW (1980) Immunological aspects of aging: An overview. In Biological Mechanism in Aging, pp. 455-467 [RT Schimke, editor]. Bethesda, MA: National Institutes of Health.

Tappia PS, Man WJ \& Grimble RF (1995) Influence of unsaturated fatty acids on the production of tumor necrosis factor and interleukin- 6 by rat peritoneal macrophages. Molecular and Cellular Biochemistry 143, 89-98.

VanMeter AR, Ehringer WD, Stillwell W, Blumenthal EJ \& Jenski LJ (1994) Aged lymphocyte proliferation following incorporation and retention of dietary omega-3 fatty acids. Mechanisms of Ageing and Development 75, 95-114.

Wander RC, Du S-H, Ketchum SO \& Rowe KE (1996) $\alpha$-Tocopherol influences in vivo indices of lipid peroxidation in 
postmenopausal women given fish oil. Journal of Nutrition 126, 643-652.

Wander RC, Hall JA, Gradin JL, Du S-H \& Jewell DE (1997) The ratio of dietary $(n-6)$ to $(n-3)$ fatty acids influences immune system function, eicosanoid metabolism, lipid peroxidation, and vitamin $E$ status in aged dogs. Journal of Nutrition 127 , $1198-1205$.

Webb DR, Rogers TJ \& Nowowiejski E (1980) Endogenous prostaglandin synthesis and the control of lymphocyte function. Proceedings of the New York Academy of Sciences USA 332, 260-270.
Whelan KS, Broughton B, Lokesh B \& Kinsella JE (1991) In vivo formation of leukotriene $E_{5}$ by murine peritoneal cells. Prostaglandins 41, 29-41.

Wu D, Meydani SN, Meydani M, Hayek MG, Huth P \& Nicolosi RJ (1996) Immunological effects of marine- and plant-derived ( $n$-3) polyunsaturated fatty acids in non-human primates. American Journal of Clinical Nutrition 63, 273-280.

Zoschke DC \& Messner RP (1984) Suppression of human lymphocyte mitogenesis mediated by phagocyte-released reactive oxygen species, comparative activities in normal and chronic granulomatous disease. Clinical Immunology and Immunopathology 32, 29-40.

(C) Nutrition Society 1998 